\title{
Reflets
}

Revue d'intervention sociale et communautaire

\section{L'influence de l'insécurité linguistique sur le parcours doctoral d'une jeune femme acadienne : une expérience teintée de la double minorisation}

\section{Tina Desabrais}

Volume 16, numéro 2, 2010

L'intervention sociale en contextes minoritaires : penser la complexité et la multiplicité des processus de minorisation

URI : https://id.erudit.org/iderudit/1000314ar

DOI : https://doi.org/10.7202/1000314ar

Aller au sommaire du numéro

Éditeur(s)

Reflets, Revue d'intervention sociale et communautaire

ISSN

1203-4576 (imprimé)

1712-8498 (numérique)

Découvrir la revue

Citer cet article

Desabrais, T. (2010). L'influence de l'insécurité linguistique sur le parcours doctoral d'une jeune femme acadienne : une expérience teintée de la double minorisation. Reflets, 16(2), 57-89. https://doi.org/10.7202/1000314ar

\section{Résumé de l'article}

Une doctorante acadienne partage son expérience à titre de femme francophone de milieu minoritaire inscrite aux études doctorales, soit une expérience de double minorisation, de même que les rapports de force, de domination et de minorisation qui accompagnent cette expérience. Cet article présente plus spécifiquement l'influence de l'insécurité linguistique,

provoquée en très grande partie par ce processus de double minorisation, sur le parcours doctoral de cette jeune femme.
Tous droits réservés @ Reflets, Revue d’intervention sociale et communautaire, 2010
Ce document est protégé par la loi sur le droit d'auteur. L'utilisation des services d'Érudit (y compris la reproduction) est assujettie à sa politique d'utilisation que vous pouvez consulter en ligne. 


\section{L'influence de l'insécurité linguistique sur le parcours doctoral d'une jeune femme acadienne : une expérience teintée de la double minorisation}

\section{Tina Desabrais}

Doctorante, Faculté d'éducation, Université d'Ottawa

\section{Résumé}

Une doctorante acadienne partage son expérience à titre de femme francophone de milieu minoritaire inscrite aux études doctorales, soit une expérience de double minorisation, de même que les rapports de force, de domination et de minorisation qui accompagnent cette expérience. Cet article présente plus spécifiquement l'influence de l'insécurité linguistique, provoquée en très grande partie par ce processus de double minorisation, sur le parcours doctoral de cette jeune femme.

Mots clés : francophonie minoritaire, femmes aux études supérieures, insécurité linguistique

\footnotetext{
Abstract

An Acadian $\mathrm{PhD}$ candidate shares her experience as a francophone woman having lived in a double minority context as well as the
} 
power-influenced relationships and the domination that come along with this experience. This article presents more specifically how linguistic insecurity, largely shaped by her experience in both minorities, has had an effect on the doctoral journey of this young woman.

Key words : francophone minorities, women in higher studies, linguistic insecurity

\section{Introduction}

"Il s'avère donc important, dans ce dernier contexte, d'étudier à la fois les sentiments et attitudes envers la langue héritée de même que les rapports de sexe, et ce, afin de rendre positive l'expérience des études supérieures en français en milieu francophone minoritaire. »
La promotion de l'ouverture à l'Autre s'accompagne parfois de mouvements de peur, du repli sur soi, voire du rejet total de l'Autre à travers des actes de discrimination. Mais qu'en est-il lorsqu'advient un rejet - ou une incompréhension — de Soi, c'est-à-dire de son propre groupe, comme cela arrive parfois au sein des communautés francophones en situation minoritaire? Ce phénomène peut surgir dans divers contextes et celui de l'éducation n'en est pas plus à l'abri, bien qu'il se dise ou se veuille démocratique et inclusif. Il s'avère donc important, dans ce dernier contexte, d'étudier à la fois les sentiments et attitudes envers la langue héritée de même que les rapports de sexe, et ce, afin de rendre positive l'expérience des études supérieures en français en milieu francophone minoritaire. Cela contribuerait à l'égalité entre les sexes et à l'engagement culturel, intellectuel et économique des francophones au sein de la société canadienne, ce qui ne peut être que bénéfique pour l'éducation universitaire en milieu francophone minoritaire canadien et ipso facto pour les communautés francophones minoritaires. C'est dans cette visée qu'une analyse microsociologique sera présentée, celle du cas d'Yvette ${ }^{1}$ (pseudonyme), une doctorante issue d'un milieu francophone minoritaire. Plus spécifiquement, il sera question de voir comment son expérience doctorale est teintée de double minorisation - francophone issue de milieu minoritaire et femme inscrite aux études supérieures — de même que de peur, de repli sur soi et d'actes de discrimination. 
Nous définirons d'abord l'insécurité linguistique. Puis, nous illustrerons comment l'expérience des femmes en milieu universitaire diffère généralement de celle des hommes et nous montrerons comment ces concepts s'articulent dans une problématique de recherche. L'approche méthodologique qui a permis de mieux comprendre le cas d'Yvette $^{1}$ sera ensuite présentée, de même que les données retenues. Elles seront suivies de l'articulation entre l'insécurité linguistique et les rapports sociaux dans l'expérience doctorale. Quelques pistes de réflexion seront enfin proposées.

\section{Le concept d'insécurité linguistique}

"L'insécurité

linguistique s'imbrique

dans des stratégies

identitaires, éclairées ou

instinctives (Taboada-

Leonetti, 1999),

tant sur le plan des

réponses stratégiques

identitaires que sur

celui des tactiques

employées ou des

"réponses »."
L'insécurité linguistique s'imbrique dans des stratégies identitaires, éclairées ou instinctives (Taboada-Leonetti, 1999), tant sur le plan des réponses stratégiques identitaires que sur celui des tactiques employées ou des "réponses $»^{2}$. De fait, les études portant sur l'insécurité linguistique soutiennent qu'elle est en grande partie attribuée à la surveillance et à la correction de la langue par ceux et celles qui possèdent la langue dominante (Labov, 1976; Bourdieu, 1982; Swiggers, 1993). Reconnaissant en pratique sinon la surveillance des "dominants " du moins la légitimité de la langue dominante (Bourdieu, 1982), les "dominés » adopteraient consciemment ou non maintes stratégies identitaires pour contrer l'exclusion, entre autres, le rejet ou la négociation de l'identité qui leur a été assignée, l'assimilation au majoritaire ou le déni. L'insécurité linguistique serait donc «la manifestation d'une quête de légitimité linguistique, vécue par un groupe social dominé, qui a une perception aiguisée tout à la fois des formes linguistiques qui attestent sa minorisation et des formes linguistiques à acquérir pour progresser dans la hiérarchie sociale" (Francard, 1997, p. 171). Pour Calvet (1993), " [o]n parle de sécurité linguistique lorsque, pour des raisons sociales variées, les locuteurs ne se sentent pas mis en question dans leur façon de parler, lorsqu'ils considèrent leur norme comme la norme » (p. 50). À l'inverse, "[i]l y a insécurité linguistique lorsque les locuteurs considèrent leur façon de parler comme peu valorisante et ont en tête un autre modèle, 
"...il est important de noter que

l'insécurité linguistique s'amplifie avec le nombre d'années d'études à temps plein, alors qu'on pourrait s'attendre à ce qu'elle s'amenuise, entre autres, en raison de l'acquisition de connaissances et de la possibilité de progresser dans la hiérarchie sociale..." plus prestigieux, mais qu'ils ne pratiquent pas » (p. 50). Bretegnier (1999) explique pour sa part que

[1] e sentiment d'[insécurité linguistique] apparaît comme lié à la perception, par un (groupe de) locuteur(s), de l'illégitimité de son discours en regard des modèles normatifs à l'aune desquels, dans cette situation, ont évalué les usages; et partant, à la peur que ce discours ne délégitime à son tour, ne le discrédite, ne le prive de l'identité, à laquelle il aspire, de membre de la communauté qui véhicule ce modèle normatif. (p. 9)

Bretegnier définit plus précisément trois types d'insécurité linguistique : normative, communautaire et identitaire. Cette dernière, à laquelle se réfere majoritairement cet article, nait de la perception par le locuteur de sa propre illégitimité en tant que membre socialement admis dans une microcommunauté linguistique donnée et par conséquent, en tant que locuteur de la variété qui y correspond.

Dans le cadre de cet article, il est important de noter que l'insécurité linguistique s'amplifie avec le nombre d'années d'études à temps plein, alors qu'on pourrait s'attendre à ce qu'elle s'amenuise, entre autres, en raison de l'acquisition de connaissances et de la possibilité de progresser dans la hiérarchie sociale (Francard, 1997). En fait, plus on étudierait, plus on prendrait conscience des différences régionales entre les discours, les dialectes régionaux et le français standard, ce qui n'a pas manqué de se produire chez Yvette. Une étude effectuée en France soulève plus précisément que « $[\ldots]$ l'institution scolaire dans le monde francophone accroîtrait l'insécurité linguistique en développant à la fois la perception des variétés linguistiques régionales et leur dépréciation au profit d'un modèle mythique et inaccessible " (Francard, cité par Moreau, 1997, p. 172).

Par ailleurs, des études effectuées à Norwich, en Angleterre, montraient dès 1972 que l'insécurité linguistique est plus élevée chez les femmes que chez les hommes. À titre d'exemple, les femmes auraient plus tendance à signaler qu'elles ont mal 
prononcé tel ou tel mot (Trudgill,1972,1998). Quelques années plus tard, en France cette fois, les observations de Bourdieu (1982) abondent dans le même sens : l'insécurité linguistique est plus élevée chez les femmes des classes moyennes, compte tenu de la division du travail entre les sexes qui fait que les femmes visent l'ascension sociale. Leurs capacités de production symbolique les voueraient ainsi, de façon générale, à investir davantage dans l'acquisition des dispositions légitimes (p. 29).

Alors que plus d'une vingtaine d'années se sont écoulées depuis les études de Trudgill et Bourdieu, on pourrait croire que la différence du degré d'insécurité linguistique ne serait plus aussi remarquable entre hommes et femmes étant donné - si l'on s'en tient aux explications offertes par ces chercheurs - qu'une majorité de femmes travaillent désormais hors du foyer familial et que plus de femmes que d'hommes étudient au niveau du baccalauréat (Robbins et Olivier, 2006). L'étude de Singy (2004), dont le champ d'observation est fixé en Suisse romande, se fonde d'ailleurs sur des hypothèses qui, contrairement à celles des auteurs sus mentionnés, insistent non pas sur des différences sur le plan

"...une majorité de femmes pensent que de manière générale il est plus important de "bien parler" pour une femme que pour un homme... l'insécurité linguistique s'exerce aujourd'hui encore de manière plus forte sur les femmes que sur les hommes..." linguistique, mais sur des similitudes entre hommes et femmes (p. 5). À la suite d'une stratégie d'observation reposant sur la saisie de discours épilinguistiques suscités par le biais d'entretiens semidirigés, Singy relève qu'une majorité de femmes pensent que de manière générale il est plus important de "bien parler " pour une femme que pour un homme (p.175). Confronté à ses hypothèses, il conclut que l'insécurité linguistique s'exerce aujourd'hui encore de manière plus forte sur les femmes que sur les hommes (p. 178), rejoignant ainsi les propos des autres chercheurs.

Bref, l'insécurité linguistique, chez les "dominés " d'abord, s'avère un facteur de minorisation. Et encore plus chez les femmes qui, dans le contexte de l'éducation, font face à un autre facteur de minorisation, soit celui d'être femme en milieu universitaire.

\section{Femmes et milieu universitaire}

Le fait d'être femme en milieu universitaire constitue en soi un facteur de minorisation, ce qui explique sans doute pourquoi 
"Bien que le nombre de femmes inscrites au premier et au deuxième cycle ait considérablement augmenté, les hommes sont aujourd'hui toujours plus nombreux que les femmes à obtenir un diplôme doctoral, représentant 58,1\% des effectifs canadiens en 2003-2004...» il fut étudié par maints chercheurs. Aux fins de cet article, ce facteur sera abordé sous deux angles, celui du nombre et celui du traitement différentiel.

Soulignons d'abord que la majorité des Canadiennes ayant entrepris des études de baccalauréat et de maitrise choisissent de ne pas faire d'études doctorales (Mujawamariya et Sethna, 2005; Robbins et Olivier, 2006). Bien que le nombre de femmes inscrites au premier et au deuxième cycle ait considérablement augmenté, les hommes sont aujourd'hui toujours plus nombreux que les femmes à obtenir un diplôme doctoral, représentant $58,1 \%$ des effectifs canadiens en 2003-2004 (Robbins et Olivier, 2006). Les femmes sont donc toujours moins nombreuses que les hommes à entreprendre une carrière universitaire - 40,7\% comparativement à 59,3\%, soit un écart de presque $20 \%$ (Robbins et Olivier, 2006) - ce qui, étant donné une population étudiante universitaire majoritairement composée de femmes, témoigne d'un problème de représentativité et perpétue une inégalité. Selon Vezina (1998), " [n]owhere are the effects of barriers more pronounced than at the master's and doctoral levels " (p. 83). Elle soulève, entre autres, le fait que "women students face barriers to their success in the curriculum, where their contribution to the literature are relatively ignored or trivialized when discussed" (p. 83) et que " the underrepresentation of women faculty members who serve as advisors, role models and/or mentors for women also acts as a barrier to these students" (p. 83). Chez les francophones de milieu minoritaire, en l'occurrence l'Ontario, lieu d'études d'Yvette, notons qu'une part plus élevée de femmes que d'hommes a fréquenté l'université - 47,3\% comparativement à $42,4 \%{ }^{3}$. Le nombre de femmes francophones qui s'inscrivent aux études supérieures en Ontario est cependant nettement inférieur à celui de leurs collègues masculins - 34,2\% pour les femmes, comparativement à $41,1 \%$ pour les hommes (Office des affaires francophones, 2005).

Outre cette minorisation qui s'observe sous une loupe quantitative, maints facteurs de minorisation sont observables sous une loupe qualitative, c'est-à-dire dans le quotidien, dans le nondit. D'ailleurs, dès 1987, Smith présentait une critique à teneur féministe et une alternative à la sociologie standard, la sociologie 
établie permettant, à son avis, une observation de la société et des relations sociales, mais ne tenant pas compte du quotidien dans lequel les gens vivent en général (p. 2). À l'époque, Smith qualifiait le monde universitaire de "male world in its assumptions, its language, its patterns of relating" (p. 7) et déplorait le fait qu' "among the most important of these boundaries, $[\ldots]$ is that which reserves to men control of the policy-making and decision-making apparatus in the educational system" (p. 26). À sa lecture, on pourrait croire que cette étude représentait la réalité du temps - elle date de plus de vingt ans - et que les résultats actuels ne seraient pas aussi notables. Un ouvrage un peu plus récent partage cependant l'avis de Smith :

Post-secondary education is irrefutably biased: it teaches and supports a male perspective of the world operates in a manner amenable to men's priorities, and the status quo continues as masculine privilege is protected and perpetuated. Excuses for the discrimination vary in creativity and credibility, reflecting either intentional illusion of academic delusion. (Stalker et Prentice, 1998, p. 29)

L'étude de Ouellette (1999) souligne à son tour un malaise, davantage relié à l'apprentissage universitaire, qui est détaché de la vie de l'apprenante, qu'à la structure administrative. À son avis, il serait particulièrement utile pour bien des femmes de mettre leur expérience en rapport avec les connaissances transmises, car les réalités qu'elles vivent sont parfois occultées par un système axé sur des préoccupations essentiellement masculines. Selon Ouellette (1999), le fait que la socialisation des filles met l'accent sur les rapports humains et sur la sensibilisation aux besoins des autres influerait sur les manières de penser et de raisonner des femmes tout au long de leur vie. Elle soutient que "penser comme une femme " correspond généralement à l'image de la pensée féminine stéréotypée, c'est-à-dire émotionnelle, intuitive et subjective (p. 98). À son avis, dans le but de mieux intervenir auprès des apprenantes adultes, il faudrait comprendre les forces sociales négatives qui risquent d'appauvrir chez les femmes leur estime de soi, de nuire à leur performance en milieu universitaire 
et d'agir sur leur identité d'apprenante — marquée par l'identité linguistique dans le cadre de cet article.

À la lumière de ces deux facteurs de minorisation, il serait possible de dire que les femmes francophones de milieu minoritaire, plus propices à ressentir de l'insécurité linguistique, sont exposées à la potentialité de vivre une expérience de double minorisation une fois en milieu universitaire, ce que précisera la problématique.

\section{Problématique}

"Il vise à mieux saisir la réalité et les intérêts de cette femme qui appartient à un groupe minoritaire et qui vit en milieu linguistique minoritaire, et à mieux comprendre les ajustements de relation lorsque s'entremêlent cette pluralité d'identités et celle de rapports de force, et ce, dans un angle d'analyse qui gravite entre une perspective interactionniste et une critique." "
Les études portant sur l'insécurité linguistique et la situation des femmes en milieu universitaire et qui font état d'une double minorisation - provenir d'un groupe linguistiquement minoritaire et être une femme inscrite aux études supérieures peuvent servir de canevas de départ à un examen de l'expérience universitaire des femmes de milieu francophone minoritaire au Canada. Selon Dewey (1968), "[...] il existerait une relation intime et nécessaire entre les processus de l'expérience et de l'éducation » (p. 61). Notre recherche de doctorat, en cours de rédaction, porte spécifiquement sur les expériences en lien avec l'(in)sécurité linguistique de femmes de milieux francophones minoritaires canadiens inscrites aux études supérieures. Cette recherche vise donc à voir comment se vit subjectivement cette double minorisation, quels en sont les effets sur la façon dont les étudiantes inscrites aux études supérieures perçoivent leur expérience et quel est son impact sur leur performance. Le présent article présente pour sa part les données recueillies auprès de l'une de vingt participantes. Il vise à mieux saisir la réalité et les intérêts de cette femme qui appartient à un groupe minoritaire et qui vit en milieu linguistique minoritaire, et à mieux comprendre les ajustements de relation lorsque s'entremêlent cette pluralité d'identités et celle de rapports de force, et ce, dans un angle d'analyse qui gravite entre une perspective interactionniste et une critique. Les enjeux pour 
l'action politique locale, mais aussi provinciale et régionale, voire nationale, seront enfin soulevés. Soulignons d'ores et déjà que les concepts présentés ci-dessus sont également vécus dans le cadre de la francophonie minoritaire canadienne, et non seulement par les femmes de ce groupe.Toujours dans le contexte de l'éducation, la recherche sur les projets d'avenir des finissants d'écoles de langue française en milieu minoritaire au Canada, menée par Allard, Landry et Deveau (2009) soutient

[qu']il serait nécessaire de mener d'autres recherches auprès de ces élèves pour étudier plus à fond la constellation des raisons de leurs choix. Les motifs qui entrent en jeu sont nombreux et complexes. Il faudrait explorer en profondeur, entre autres, les questions suivantes: les attitudes envers la langue française, l'insécurité linguistique qui peut être plus importante dans certaines régions, la relation entre la langue des études et la langue de travail anticipée, la distance plus considérable à parcourir pour suivre un programme dans un établissement francophone et l'interaction entre coûts et distance. (p. 138)

L'éducation étant à notre avis le berceau de la culture et de l'identité, il est important pour les communautés francophones minoritaires canadiennes en général d'étudier les attitudes envers la langue française, l'insécurité linguistique et la relation entre la langue des études et la langue de travail anticipée, ce pour quoi cette étude fut menée. Le cas d'Yvette y est relativement exemplaire. Nous l'avons donc retenu aux fins de cet article, et ce, en raison de l'intensité et de la richesse des propos de l'étudiante, et aussi de la clarté avec laquelle elle partage son expérience de double minorisation. Cela dit, voyons la procédure retenue pour recueillir ses propos et ceux des autres participantes.

\section{Méthodologie}


"Le protocole de Seidman (2006) qui se divise en trois entrevues distinctes avec chacun des participants - le recueil du passé du participant et du contexte de ses expériences passées; l'interrogation sur son expérience actuelle; le sens accordé à ces expériences et la signification qu'il $y$ attribue - a inspiré une telle description en profondeur."
Cette recherche fut réalisée en faisant appel à des étudiantes de milieux francophones minoritaires inscrites à des études de maitrise ou de doctorat et recrutées au moyen d'affiches apposées sur les murs d'une université bilingue qui accueille des francophones de partout au pays. Il s'agit donc d'échantillons volontaires (voluntary samples) et d'échantillonnage boule de neige (snowball sampling) (Angers, 2005, p. 110) assurés par le réseau personnel de l'auteure ou par le biais de participantes.Vingt étudiantes ont été recrutées, onze inscrites à la maitrise et neuf au doctorat. La recherche visait à mieux comprendre l'expérience des études supérieures telle que vécue et décrite par les participantes, tout en accordant une priorité au concept d'(in)sécurité linguistique possiblement ressentie au cours de leurs études. La stratégie principale de la collecte de données fut l'entretien semi-structuré, ou semi-dirigé (Savoie-Zajc, 2006). Ce type d'entretien a permis de dégager une riche compréhension du phénomène. Le protocole de Seidman (2006) qui se divise en trois entrevues distinctes avec chacun des participants - le recueil du passé du participant et du contexte de ses expériences passées; l'interrogation sur son expérience actuelle; le sens accordé à ces expériences et la signification qu'il y attribue - a inspiré une telle description en profondeur. Comme " [...] nous vivons, du berceau à la tombe, dans un monde de personnes et de choses qui, dans une large mesure, est ce qu'il est à cause de ce qui a été fait et transmis à la génération présente par les activités antérieures » (Dewey, 1968, p. 85), une première entrevue individuelle visait d'abord à recueillir le contexte des expériences passées de la participante, puis à l'interroger sur son expérience actuelle. Une seconde entrevue a ensuite permis d'interroger les participantes sur le sens qu'elles accordent à leurs choix et à leurs expériences, de même que sur la signification qu'elles leur attribuent. Finalement, une entrevue de groupe fut menée, considérant que ce type d'entrevue procurerait l'occasion de «mettre en commun et d'éclairer les compréhensions respectives en regard du phénomène étudié » (Deschamps, 1993, p. 61). Ce groupe de discussion a permis à dix participantes, dont Yvette, d'échanger librement sur leur expérience aux études supérieures, à la fois à titre de femme et de membre d'un groupe linguistique 
minoritaire, en d'autres mots, à titre de double minorité. Le groupe fut mené par l'auteure de la recherche et des thèmes et questions ont orienté la discussion, comme cela fut fait lors des entretiens semi-dirigés.

Après avoir collecté et transcrit les données provenant des entretiens, l'analyse a débuté par la lecture de l'ensemble des données dans le but d'en tirer le sens global, puis une classification inspirée d'une démarche d'analyse inductive générale privilégiée. Cette démarche est « définie par un ensemble de procédures systématiques permettant de traiter des données qualitatives, ces procédures étant essentiellement guidées par les objectifs de recherche " (Thomas, 2006, cité par Blais et Martineau, p. 3). Comme le soulignent les auteurs, l'objectif principal est de développer des catégories de données. À leur avis, l'idéal serait en fait de créer un nombre restreint de catégories, entre trois et huit, " afin que le chercheur puisse avoir une vue d'ensemble des aspects clés qui ont été identifiés dans les données brutes et qui sont considérés comme étant les plus importants, étant donné les objectifs de recherche visés " (p. 8$)^{4}$. Pour mettre en lumière la complexité de la double minorisation, cet article se limite à présenter les propos relatifs à l'identité, à l'insécurité linguistique et au statut de femme en milieu universitaire. La présentation d'un cas spécifique permet également de traiter le sujet plus en profondeur et de vivre plus intimement l'impact du processus de double minorisation. Pour ce faire, un portrait d'Yvette est d'abord esquissé, suivi du regard qu'elle pose sur son appartenance à un milieu francophone minoritaire et sur son expérience de femme inscrite aux études doctorales.

\section{Portrait d'Yvette}

Originaire d'un milieu rural majoritairement francophone dans l'est du Nouveau-Brunswick et étudiante au doctorat,Yvette avait 24 ans au moment de l'entretien. Elle avait un conjoint et n'avait pas d'enfant à sa charge. Sa langue parlée à la maison était le français et elle étudiait également en français. Elle est une étudiante de 
première génération (ÉPG) (Horn et Nu ez, 2000; Choy, 2001; McCarron et Inkelas, 2006; Engle, 2007). Elle a d'abord fait des études de baccalauréat au Nouveau-Brunswick, puis elle est allée étudier en France pour compléter une maîtrise en science politique et, a-t-elle dit, pour perfectionner son français. Ensuite, elle est revenue au pays pour y poursuivre en français des études doctorales. Elle se dit francophone et Acadienne.

\section{Appartenance à un groupe linguistique minoritaire}

"Mais le fait d'être francophone prend une tournure plus complexe lorsque les francophones eux-mêmes se "délégitimisent», se jugent entre eux ou encore perçoivent cette partie de leur identité comme étant une parmi tant d'autres. "

\section{L'identité francophone}

Symbole de l'identité, la langue est le plus puissant facteur d'appartenance sociale et ethnique et à la fois, un facteur de différenciation et d'exclusion (Leclerc, 2007). Les propos d'Yvette abondent dans ce sens : «Pour moi, quand on parle de français, pour moi c'est vraiment quelque chose d'identitaire " (Entretien 1, 5 juin 2008). Mais le fait d'être francophone prend une tournure plus complexe lorsque les francophones eux-mêmes se "délégitimisent ", se jugent entre eux ou encore perçoivent cette partie de leur identité comme étant une parmi tant d'autres. Cette "assimilation au majoritaire " est une stratégie identitaire définie par Taboada-Leonetti (1999). Elle consiste à nier ou à se débarrasser d'une identité minoritaire infériorisée en se désolidarisant de son groupe d'appartenance ou en refusant cette appartenance pour chercher à pénétrer dans le groupe majoritaire, anglophone en l'occurrence.

On peut observer ce processus dans les propos d'Yvette :

Yvette : J'ai fait mon année en France puis là, j’suis retourné en Acadie. Pis, j'me suis dit : qu'est-ce qu'on fait? Qu'est-ce qu'on fait? S'identifier en tant que francophone alors qu'on n'a pas la meilleure connaissance de la langue, alors que pas tout le monde peut bien fonctionner en français... Donc, pourquoi on revendique quelque chose qu'on ne fonctionne pas très bien dedans, tsé là. Fait que moi,j'en ai 
"Le désillusionnement suivant le séjour d'Yvette en France est en fait si fort qu'elle doute de la légitimité des siens de se considérer comme francophones et ressent de la rancoeur à l'égard de son héritage linguistique : J'en ai voulu un peu à ça aussi ». On voit ici toute l'importance attribuée à la norme et au "bon français", de même que le heurt symbolique entre le "nouveau parler" qu'elle a adopté et celui, inchangé, de son entourage." voulu un peu à ça aussi, tsé. Quelqu'un qui me reproche de bien parler français ben ah oui tsé... Tsé si j'veux m'dire francophone en quelque part, j'ai les droits de vouloir tsé m'adapter à cette norme-là même si je sais pas. Moi, j'pense que c'est plus moi qui me fais juger que moi juger, parce que c'est vraiment un rapport avec moimême. Fait que j'pense que quelqu'un pourrait critiquer ben oui si tu fais ce changement-là, c'est parce qu'en quelque part tu critiques ce registre-là. (Entretien 2, 17 juin 2008)

Ces propos ne sont pas sans rappeler Les exclus de l'intérieur de Bourdieu et Champagne (1992). Le désillusionnement suivant le séjour d'Yvette en France est en fait si fort qu'elle doute de la légitimité des siens de se considérer comme francophones et ressent de la rancœur à l'égard de son héritage linguistique : "J'en ai voulu un peu à ça aussi ». On voit ici toute l'importance attribuée à la norme et au "bon français ", de même que le heurt symbolique entre le "nouveau parler " qu'elle a adopté et celui, inchangé, de son entourage. Par ailleurs, selon Yvette, c'est ici les tenants de la langue vernaculaire qui portent un jugement à son parler, plus standard, que l'inverse, ce qui s'explique possiblement par le fait qu'ils assument dans ce contexte le statut majoritaire. Soulignons de plus le passage où elle affirme : «C'est vraiment un rapport avec moi-même ». Elle met certes des efforts pour améliorer sa langue parlée pour son propre avantage. L'on pourrait toutefois douter que le rapport soit réellement relié à elle-même : si l'on tient à modifier, à " améliorer » la façon dont on parle, c'est par référence à un Autre, à un récepteur qui pourra percevoir notre accent, notre articulation, notre débit ou notre vocabulaire. Le besoin serait nul de porter une attention à son parler si personne n'était présent pour le constater et inévitablement le juger, que ce soit positivement ou négativement. Quoi qu'il en soit, selon Yvette, la langue est une partie essentielle de l'identité :

Yvette :Je peux dire que pour moi la langue est toujours là... C'est toujours là à chaque jour... 
Fait que, ça m'habite beaucoup

Nous : Je vois ça! C'est la première fois que je demande à quelqu'un : "parle-moi de toi ", puis qu'il me parle seulement de la langue!

Yvette : Ouais! Pour moi, c'est vraiment central. Puis, je pense que c'est vraiment le FIL DIRECTEUR de tout le reste. (Entretien 1, 5 juin 2008)

«...[l]e positionnement discursif des acteurs sociaux laisse [...] entrevoir une francophonie acadienne qui s' "hétérogénise " et qui [...] prend part à une diversification et à une pluralisation de son espace sans faire fi du lieu à partir duquel la parole est prise. "
Non seulement la langue française aide cette participante à définir qui elle est, la culture acadienne joue également ce rôle.

\section{L'identité acadienne}

Selon Traisnel et Violette (2010), « [1]e positionnement discursif des acteurs sociaux laisse [...] entrevoir une francophonie acadienne qui s' "hétérogénise " et qui [...] prend part à une diversification et à une pluralisation de son espace sans faire fi du lieu à partir duquel la parole est prise» (p. 118).

On peut bien observer ce positionnement discursif dans les propos d'Yvette. Lorsqu'on lui a demandé de parler d'elle-même, elle a répondu :

Yvette : Nous en Acadie, on a... genre... une fierté acadienne. Pis, j'me rends compte comment précieux qu'c'est pour l'identité, parce que j’pense que ça m'empêche de m'poser plein de questions, tsé! Clairement, je souffre de toutes les parties d'insécurité linguistique, mais en même temps, j'ai une fierté incroyable de mon identité [...]Tsé! on a un discours qui va avec; on a un vécu qui va avec... Puis,j’pense que ça vient d'une histoire politique de revendication [...] Tu vois... Donc, souvent on s'dit : ben là! c'est parce qu'on devrait connaitre autre chose. Donc, tsé! on a quand même nos limites, mais quand ça vient à notre propre identité, moi j’pense 
que c'est assez clair. Mais j'étais pas, tsé... Mais j'suis sortie de là en m'disant : OK! tsé! au-moins moi, c'est clair au niveau identitaire acadienne francophone, tout ça, ça va. (Entretien 2, 17 juin 2008)

Même s'il paraît "clair " pour elle qu'elle est Acadienne et francophone et que cela lui évite d'avoir à se poser des questions quant à son identité, elle affirme au cours du même entretien :

Yvette : C'est difficile de s'dire oui! j'suis francophone! Mais en même temps, j'ai de l'influence de l'anglais. Puis, tsé!, à la fois de s'réconcilier avec notre identité, puis de dire... Je sais pas, mais j'me suis rendue compte que c'est hyper complexe encore aujourd'hui. (Entretien 2, 17 juin 2008)

Effectivement. Cela explique sans doute pourquoi les commentaires d'Yvette se rapportent plutôt à l'insécurité linguistique identitaire (Bretegnier, 1999) :

Yvette :J'ai eu tout un questionnement au sujet de mon identité parce que là, j'étais un peu fầchée qu'on parlait mal au Nouveau-Brunswick. Parce que je me disais... moi j'arrive ICI [à Ottawa] puis je me dis francophone, puis j'ai de la difficulté avec ma PROPRE langue... Puis je suis repartie au Nouveau-Brunswick en me disant : c'est quoi cette revendication-là qu'on fait en tant que francophones hors Québec? En tant que francophones POINT? Qu'on n'est même pas capables de maitriser notre langue qu'on veut défendre. (Entretien 1, 5 juin 2008)

On perçoit ici très bien les réactions fortement négatives soulevées par Labov (1976) envers la façon héritée de parler, réactions qui, dans le cas d'Yvette, sont si fortes qu'elle remet même en question l'identité linguistique des gens de son milieu 
"On perçoit ici très bien les réactions fortement négatives soulevées par Labov (1976) envers la façon héritée de parler, réactions qui, dans le cas d'Yvette, sont si fortes qu'elle remet même en question l'identité linguistique des gens de son milieu — " en tant que francophones POINT » »
— « en tant que francophones POINT». Au cours du second entretien, elle ajoute :

Yvette : J'regarde Antonine Maillet... Antonine Maillet a parle pas comme... comme elle écrit, euh, La Sagouine puis tout ça... J'veux dire, c'est pas possible, tsé là! ... Fait qu'un moment donné, c'est... non c'est quelque chose que j'tiens à. Pis même en fait, euh, on parlait des enfants là, pis si jamais j'aurais des enfants, j'pense que j'voudrais vivre en France, parce que moi je tiens à c'que si JAMAIS... J'ai pas cette envie maternelle-là, mais des fois $j$ 'pense comme, tsé ben, si jamais ça arrive là, mais si jamais ça arrive, je l'sais pas... J'ai l'impression qu'ils apprendraient déjà le chiac avec le contact familial, tsé, avec les cousins et tout ça. Moi, j'aurais... j'aurais tendance à leur donner une base plus forte en français. Pour moi, ça, ça voudrait dire que j'fais un effort pour habiter en France... Fait que j'suis peutêtre, j’suis peut-être un peu extrémiste dans ça, mais je... je sens vraiment le besoin. Parce que je vois des gens de l'Acadie qui ne peuvent pas fonctionner en français, pis je juge que c'est inacceptable. (Entretien 2, 17 juin 2008)

Ainsi, elle irait jusqu'à déménager dans un autre pays pour permettre à ses enfants d'apprendre à parler un «bon » français, du moins un français jugé comme étant meilleur que celui que l'on parle au Canada, et plus précisément au Nouveau-Brunswick. En ce qui a trait au chiac ${ }^{5}$, il s'apprendrait, selonYvette, naturellement avec le contact familial des cousins. Est-il réaliste de croire qu'un enfant qui grandit en France et qui vient visiter sa famille néobrunswickoise hypothétiquement une fois par année « apprendra » tout simplement le chiac avec toutes les teintes, les subtilités et les sentiments rattachés aux termes et aux accents qui y sont rattachés? Quoi qu'il en soit, aux dires d'Yvette, il semblerait que le chiac "s'attraperait ", si l'on peut dire, tout naturellement au contact 
"Un point à soulever chez la majorité d'entre elles, toutefois, est le fait qu'elles ressentent surtout de la sécurité linguistique lorsque vient le moment d'écrire, ce qui ne se traduit pas à l'oral, dans tous les cas sans exception." avec les autres locuteurs, ce qui explique peut-être l'omniprésence de l'insécurité linguistique dans ces passages traitant de l'identité acadienne.

\section{L'(in)sécurité linguistique}

Bien que cette recherche porte sur l'insécurité linguistique, certaines des participantes ont partagé le fait de se sentir à l'aise avec leur langue, soit ressentir une "sécurité linguistique " (voir la définition de Calvet, en début d'article). Un point à soulever chez la majorité d'entre elles, toutefois, est le fait qu'elles ressentent surtout de la sécurité linguistique lorsque vient le moment d'écrire, ce qui ne se traduit pas à l'oral, dans tous les cas sans exception. Cette perception d'avoir une "force » dans la rédaction $\mathrm{du}$ français revient dans les propos d'Yvette :

Yvette : À L'ÉCRIT ça se passe super bien. Je ne sais pas, à l'écrit ça m'est toujours venu assez facilement, puis là, après mon année en France, comme en français là... comme même, j'ai toujours des commentaires des profs comme quoi j'écris comme, comme j'ai toujours été considérée au-dessus de la moyenne de la classe parmi les meilleurs ou tout ça. Mais non, j'ai vraiment une force là. (Entretien 1, 5 juin 2008)

Cela dit,l'on pourrait être été tenté de croire que les participantes qui connaissent une sécurité linguistique à l'écrit porteraient un jugement moins sévère à l'égard de leurs compétences à l'oral, car elles ont, de toute façon, compris le système grammatical, si l'on peut dire, et seraient conscientes de leur niveau de langue et des erreurs qu'elles font à l'oral. Cependant,

[...] le rapport à l'écrit(ure) peut être en partielle contradiction avec les pratiques réelles des mêmes sujets, puisqu'il est aussi le produit par exemple des idéologies dominantes concernant l'écrit, ou de l'histoire de vie particulière, ou 
"Ainsi, le fait de connaître une certaine aisance en rédaction ne garantit pas la sécurité linguistique, et ce, même si le fait de bien écrire a été le principal élément soulevé lorsque des participantes ont manifesté de la sécurité linguistique. » de modes de construction subjectifs du rapport plus ou moins en décalage avec les expériences. (Schneuwly, 2008, p. 138)

Les propos d'Yvette illustrent bien cette réflexion :

Yvette : Je souffre vraiment d'insécurité linguistique. Je pense que je suis très dure sur moimême, puis je suis quelqu'un qui est vraiment forte à l'écrit. Mais quand ça vient à mon parler, je m'agace moi-même... Je vais parler, puis je vais me corriger EN PARLANT, tu vois. Puis, c'est quelque chose de difficile pour moi. (Entretien 1, 5 juin 2008)

Elle ajoute plus tard :

Yvette : Quand ça vient à parler spontanément OUI. Moi, je dirais que ça me dérange comme c'est quelque chose que je vis difficilement parce que j'y pense TROP (Entretien 1, 5 juin 2008).

Ainsi, le fait de connaitre une certaine aisance en rédaction ne garantit pas la sécurité linguistique, et ce, même si le fait de bien écrire a été le principal élément soulevé lorsque des participantes ont manifesté de la sécurité linguistique. Bien au contraire,Yvette semble trouver assez pénible le fait de s'exprimer oralement, étant très consciente des termes employés par les dominants et de leurs tournures de phrases. Elle a d'ailleurs manifesté de l'insécurité linguistique lors des entretiens en ayant recours à quelques reprises à l'hypercorrection ${ }^{6}$. Elle " fige " même parfois, tellement elle souhaite bien s'exprimer tout en essayant de le faire de façon naturelle :

Yvette : On dirait qu'j'arrive même pas à être assez spontanée en français parce que, tsé, tu... J'écris tellement bien que j'devrais pouvoir parler aussi bien qu'j'écris. Mais j'pense que j'suis tellement 
critique sur mon accent, sur c'que j'dis, sur tout, que j'me fige en quelqu'part. C'qui fait que ça empêche un peu la spontanéité. (Entretien 1, 5 juin 2008)

Elle partage de plus vouloir suivre un cours pour peaufiner son français. Plus spécifiquement, elle désirerait avoir une professeure à Paris - l'Autre, dominant - pour l'accompagner dans ce cheminement. À la rigueur, on peut percevoir du francocentrisme dans ses paroles, des cours de français étant également offerts, et de façon tout aussi efficace, au Canada :

Yvette : Idéalement, j'aimerais ça avoir une prof de français à Paris qui travaille avec moi, qui m'écoute oralement, qui relève mes fautes puis... Tsé,j'veux vouloir m'améliorer parce que j'ai déjà faite des cours en France, pis j'ai décidé que chaque petite erreur, ils vont te l'soulever. Pis sais-tu quoi? C'est correct ça. Mais il faut que moi j'fasse l'effort, pis j'suis prête à faire l'effort. (Entretien 2, 17 juin 2008)

Non seulement Yvette veut-elle faire l'effort d'améliorer ses compétences en français, elle souligne par ailleurs qu'elle résiste à l'étiquette «bilingue ":

Yvette : J'résiste un p'tit peu à l'étiquette «bilingue " parce que $\mathrm{j}$ 'me dis si tu peux pas être fonctionnel dans une langue ou l'autre, c'est pas vraiment une bonne idée... Fait que moi,j'pense que c'est aussi ça la problématique. Tout ça, c'est qu'on a tellement développé le bilinguisme au Canada, ben en tout cas au NouveauBrunswick, pis j'me rends compte qu'en Ontario, c'est vraiment présent, que finalement les francophones, c'est pas des anglophones qui adoptent le bilinguisme comme ça. C'est vraiment les francophones. Pis c'est clair que ça 
"Ce rapport à

l'Autre dominant, qu'il soit Français ou Québécois, joue un rôle substantiel dans ses représentations et sentiments linguistiques, de même que dans son rapport à la langue." crée de l'insécurité parce que soudainement, on sait plus c'est quoi un anglicisme. Parce que c'est TELLEMENT ancré dans notre langue que, tsé, c'est pas la même chose que les Québécois. Les Québécois peuvent te l'dire tout d'suite que c'est un anglicisme, tsé. Nous autres, ça vient, euh, par les p'tits là, pis c'est moins important pour nous parce que, pour nous, l'anglais a une place j'pense au sein du français en quelque part. (Entretien 2, 17 juin 2008)

On constate ici une généralisation : tous les Québécois ne peuvent pas toujours "dire tout d'suite que c'est un anglicisme". Songeons seulement à la locution "à date » couramment utilisée par les Québécois et qui est un calque de l'anglais up to date et qui devrait se dire "à ce jour » ou " jusqu'à maintenant ». Ce passage permet de bien voir le rapport d'Yvette aux « dominants » de la langue. Ce rapport à l'Autre dominant, qu'il soit Français ou Québécois, joue un rôle substantiel dans ses représentations et sentiments linguistiques, de même que dans son rapport à la langue.

Yvette commentera à nouveau son rapport avec les "dominants québécois", cette fois en comparaison aux « dominants français » :

Yvette : Ah! les Québécois ont toujours été TELLEMENT durs sur moi là, c'est fou tsé là. Parce que j'travaillais au centre touristique puis... Puis en tout cas, ils parlaient toujours en anglais, puis euh...

Nous : Ah oui?

Yvette : Ouais! Pis j'avais ben d'la difficulté avec ça. Mais j'me suis rendue compte que c'est pas une tendance personnelle. Les Québécois ont tendance à faire ça là avec euh beaucoup de francophones hors Québec. 
Nous : Alors t'as eu plus de difficulté avec les Québécois qu'avec les Français?

Yvette :Ah oui! avec les Français, ça va. Même que beaucoup de Français m'ont dit, tsé, c'est pas juste une expérience isolée ou une, tsé comme, beaucoup de Français m'disaient que, euh, ils me comprenaient mieux parce que j'avais pas l'accent du joual. (Entretien 2, 17 juin 2008)

Une expérience qu'elle avait partagée lors du premier entretien vient toutefois nuancer ce propos :

Yvette : J'AI EU à faire des exposés en France où est-ce que disons que $\mathrm{j}$ 'accordais mal quelque chose ou que je faisais une petite erreur, ils allaient me le dire tout de suite. Fait que là, si j’y pense trop, je ne veux plus parler. (Entretien 1, 5 juin 2008)

"...un locuteur

pourrait parfois

même être victime de rapport de pouvoir en raison de l'accent, $d u$ vocabulaire employé ou tout simplement de la "façon d'être ", et du coup voir son intégrité intellectuelle mitigée. »
On constate ici qu'outre les capacités intellectuelles ou encore les compétences requises pour bien exercer le "métier d'étudiant " (Coulon, 1993), un important facteur de réussite des études universitaires, au niveau du baccalauréat, certes, mais d'autant plus aux études supérieures, est sans contredit les compétences linguistiques, entre autres, celle de bien transmettre ses idées, voire de bien s'exprimer tout court. Sans ces dernières, la communication pourrait s'avérer infructueuse et un locuteur pourrait parfois même être victime de rapport de pouvoir en raison de l'accent, du vocabulaire employé ou tout simplement de la "façon d'être ", et du coup voir son intégrité intellectuelle mitigée. D'autres manifestations de rapport de pouvoir peuvent également être observées dans les échanges entre hommes et femmes, en contexte universitaire. 


\section{Être une femme inscrite aux études supérieures}

"...certains passages

révèlent un rapport dominant-dominé

entre les étudiantes et le corps professoral, ou encore, entre les étudiantes et leurs confrères masculins. "
De tous les milieux, le milieu universitaire devrait être celui qui se veut le plus compréhensif et le plus respectueux du choix de femmes de poursuivre des études supérieures, mais force est de constater que ce n'est pas toujours le cas : certains passages révèlent un rapport dominant-dominé entre les étudiantes et le corps professoral, ou encore, entre les étudiantes et leurs confrères masculins. Comme cette question n'a pas été posée directement, seules quelques participantes ont soulevé de telles observations. Sans être généralisables, leurs propos méritent tout de même une attention. À ce sujet, rapportons les propos d'Yvette qui, en référence à son expérience universitaire, se dit "très consciente du fait [qu'elle est] une femme » :

Yvette : J'pense que ça on s'en sort pas, tsé. Pis c'est différent, tsé, là comme... Pis des fois, c'est difficile à gérer parce que j'travaille en tant qu'un prof... hum... Comme y'était toujours plus facile avec moi, pis y m'faisait des clins d'œil, pis y'était ... Pis c'est difficile à gérer en tant que femme parce que tu t'dis OK, moi là, parce que les autres m'le disait : ah! tu devrais aller lui parler de telle chose parce qu'y est plus gentil avec toi OK. Mais en même temps, comment tu gères ça? ... Comment tu gères ça? Est-ce que... Est-ce que tu lui dis? Pas vraiment. Parce que, tsé, c'est une bonne chose pour moi, tsé. Comme moi, c'était très facile travailler avec lui, puis tout ça, en même temps, y'a une partie d'toi qui t'dit ouf! tsé, ça devrait pas être comme ça. Fait que, tsé, des fois, je j'préfêre que ça ça arrive pas, mais quand ça arrive, c'est difficile à gérer aussi... Ben, je l'sais pas vraiment. Tu veux pas non plus devenir une des ces femmes-là qui crient, euh, alerte, euh,j'me fais harceler, tsé comme... Fait 
que j'trouve qu'on a beaucoup d'bagage avec nous. (Entretien 2, 17 juin 2008)

On voit ici l'exemple de traitements de faveur et d'une relation imposée par un professeur, parce qu'elle est femme, lui semble-til. Il serait en quelque sorte tout à l'avantage d'Yvette de " jouer le jeu » et de maintenir de bons contacts avec ce professeur, mais ce conflit éthique lui pèse lourd sur la conscience — voir «le bagage » qu'elle transporte. Bien qu'elle soutienne que c'est une "bonne chose " pour elle, elle ressent tout de même un malaise. Ce dernier est trahi par la crainte d'exagérer ou d'être accusée de dramatisation si elle se prononce sur le sujet. Cette situation est délicate, car elle ne présente pas particulièrement de comportement stigmatisant, bien qu'il soit ostentatoire, et ne peut donc être reproché formellement, et ce, même s'il provoque un inconfort. Il revient donc à Yvette de le "gérer ", elle qui ne souhaiterait que d'être appréciée pour ses compétences et son rendement, au point même de vouloir se " cacher ", s' « effacer " :

Yvette : J'ai mis quinze livres [elle a pris 6,8 kilos] depuis que j'suis à la maitrise, pis je pense que c'est parce que... OK, en quelque part là,je cherchais à m'cacher. Tsé, moi j'crois beaucoup dans, comme tsé, on... on mange nos émotions pis tout ça... Pis j'avais tellement peur de passer comme, tsé, celle qui était cute ou bla bla bla que j'ai mis quinze livres pour m'effacer, pis à un moment donné, y a un gars dans ma classe qui a commencé hum genre la séduction. J'étais tellement... tellement... J'ai peut-être été trop méchante, mais j'te l'jure y a lâché l'cours. Parce que là, j'étais rendue à un point c'était comme non! non! non! non! non! non! J'suis dans une salle de classe, j'suis pas ici pour ça... Mais c'est présent. Pis là,j'me dis pour le doc, il faut que j'gère d'une autre façon là. Tsé, j'veux pas compromettre ma figure parce que y a quelques gars, tsé, qui sont, tsé, qui sentent le besoin 
d'essayer là... Mais, hum ouin... ouin... J'me suis rendue compte que ça été, euh tsé, parce que chaque fois que quelque chose comme ça m'arrivait, ben j'me... j'me réconfortais dans l'Nutella. Tu vois là...

Nous : Ah! Oui!

Yvette : Ben oui! Parce que, tsé, tu veux être reconnue pour ton intelligence... Puis... Tsé! j'pense que toutes les filles passent à travers de ça... Pis des fois, tu t'sens juste comme... Ah tsé là... Fait que des fois j'essaie de sonner deux fois plus intelligente, tsé là, comme... Tsé, j'suis très consciente des efforts que j'fais. (Entretien 2, 17 juin 2008)

Cette envie de passer inaperçue, peut-on dire, provient donc d'abord d'un traitement différentiel de la part d'un professeur, puis du fait d'avoir été courtisée en salle classe. Cela s'est produit, selon Yvette, dans un contexte inapproprié étant donné l'aspect sérieux attribué aux études supérieures, mais également, comme présenté ci-dessous, en raison du fait qu'il puisse être difficile de s'exprimer et de faire valoir ou reconnaitre son point de vue :

Yvette: Si j'arrive, pis j'dis quelqu'chose puis, tsé, j'suis très argumentative, ça passe pas bien. Mais pourtant, les gars y font la même chose, pis ça passe bien... Mais $\mathrm{j}$ 'me suis rendue compte que si je dis quelque chose, mais je l'ouvre un peu en question, tsé, comme... Comme... Ah je lisais le texte dans Brown, puis euh en tout cas j'me rendais compte que bla bla bla. Fait que j'me d'mandais c'que vous en pensiez. LÀ, ça passait toujours bien. Mais aussitôt que j'disais voici ce que je pense de bla bla bla, non ça passait pas. Ça, c'est quelque chose que j'ai trouvé. 
Nous: Quand ça passait pas, c'était quoi la réponse?

Yvette : Ben, c'tait vraiment comme les gens. J'sentais une froideur pis y a beaucoup d'gars aussi dans mes séminaires. Pis, je sentais qu'y réagissaient pas. Je sentais qu'ils voulaient pas partager, qu'ils voulaient pas dialoguer avec moi. OK! Alors que quand j'le faisais comme ça, y'avait plein d'gens qui sautaient sur c'que j'avais dit, si... si j'le faisais de manière plus ouverte. Mais sinon là... chose d'inapproprié, pis là personne n'en parlait. Mais justement, j'disais la même chose, mais juste de manière différente. Pis, j'me suis dit oui, ça c'est parce qu'on est... han tsé... en tant que femmes. Les hommes préfèrent l'entendre d'une certaine manière $[. .$.$] C'est qu'j'suis très argumentative,$ pis les hommes ça passe, mais moi parce que j'suis une femme [...] Mais j'suis attendue en tant que femme de comprendre, pis j'ai pas envie de comprendre. Parce que, tsé, pour moi c'est wow, c'est exactement c'que j'veux pas être. Tsé, on parlait de ça les... les filles disaient... y a les directeurs disaient faut laisser les émotions à la porte. Mais moi, j'fonctionne comme ça... Je... je ne veux pas être vue comme une femme, tsé, comme étant différente... Tsé, j'ai toujours lutté contre ça. C'qui fait que j'suis peut-être complètement désensibilisée aux femmes qui pleurent alors. Comme parce que j'me dis c'est correct de pleurer, on pleure. Mais fais-le pas dans l'bureau de ton prof... Si possible, tsé là... Parce que justement, ça, ça contribue au fait que on demeure des... tsé... Ah! les femmes! faut faire attention...

Nous : Sont fragiles? 
"...les femmes participent en général davantage aux discussions que les hommes, sauf lorsqu'il $y$ a présence d'un nombre supérieur d'hommes dans la classe. »
Yvette : Ouin! c'est ça! Puis ouin, ça c'est quelque chose que j'résiste là. Ouin! Tsé, en discutant, j'me rends compte que finalement, ça pis je l'sais. Mais qu'est-ce que tu vas faire... J'peux rien changer à ça, tsé. (Entretien 2, 17 juin 2008)

Ce passage est lourd de sens. À ce sujet, Cameron (1995) explique :

Recurring emphasis on the importance of "ladylike" speech manifests not only class prejudice but also a more specifically sexist logic that puts women in a curious double-bend. Tinkering with your speech through such practices as elocution is seen as a "feminine" activity precisely because it is superficial, merely cosmetic. Yet like other cosmetic practices it is often expected of women and made normative for them. One consequence of this is that the linguistic norms saying women should "mind their language " apply to some degree across the social spectrum - "correctness 》 is associated with femininity as well as with high social status. (p. 170)

Cela rejoint également les conclusions de maintes études sur le sujet, entre autres, celle de Howard et Henney (1998) et celle de Canada et Pringle (1995) qui démontrent que les femmes participent en général davantage aux discussions que les hommes, sauf lorsqu'il y a présence d'un nombre supérieur d'hommes dans la classe. Les résultats d'une récente étude sont d'autant plus étonnants puisque la recherche a été menée auprès de groupes où les femmes étaient deux fois plus nombreuses que les hommes (Kozanitis et Chouinard, 2007). Leurs résultats vont davantage dans le sens de ceux d'études qui datent des années 70 et 80, entre autres, celles de Boersma et collab. (1981) et de Sadker et Sadker (1986), qui rapportaient alors un taux de participation verbale plus élevé chez les hommes. En somme, les événements relatifs à l'environnement de la classe soulevés par 
"Cela indique que le travail à effectuer va au-delà des ressources disponibles et des moyens établis et consiste, aujourd'hui encore, à conscientiser les gens sur certains comportements ou attitudes adoptés qui peuvent s'avérer compromettants à l'égard des femmes en milieu universitaire, et encore plus à l'égard de celles inscrites aux études supérieures. » cette participante rejoignent les études de Janz et Pyke (1997) qui faisaient part, parmi d'autres facteurs, de l'impact des propos sexistes ou stéréotypés en salle de classe et sur le campus en général, de la propension de certains professeurs à agir distinctement selon le sexe des étudiants, et également de la faible représentativité de modèles féminins au sein de certaines facultés.

Les observations soulevées par ces participantes témoignent ainsi du fait que certains comportements préjudiciables seraient toujours présents dans la salle de classe de 2008 , et ce, même après tous les efforts de sensibilisation et d'éducation - songeons, entre autres, aux Women Studies -, les règlements d'équité ou les services mis en place pour mieux desservir la clientèle féminine. Cela indique que le travail à effectuer va au-delà des ressources disponibles et des moyens établis et consiste, aujourd'hui encore, à conscientiser les gens sur certains comportements ou attitudes adoptés qui peuvent s'avérer compromettants à l'égard des femmes en milieu universitaire, et encore plus à l'égard de celles inscrites aux études supérieures.

\section{Conclusion}

Des données issues d'entretiens avec l'une des étudiantes qui ont participé à une recherche doctorale portant sur l'influence de l'insécurité linguistique sur l'expérience des études supérieures ont été présentées aux fins de ce numéro de Reflets. Ses propos relatifs au fait d'être à la fois francophone de milieu minoritaire et femme inscrite aux études supérieures ont bien illustré l'expérience de la double minorisation de même que les rapports de force, de domination et de minorisation. Chez Yvette, on a pu observer plus particulièrement le dénigrement d'une partie importante de soi et de son expérience antérieure, ce qui a créé chez elle un conflit identitaire important de même que l'auto-imposition de limites conscientes ou inconscientes sur le plan de la performance - à titre d'exemple, s'exprimer moins ou différemment, ou encore figer lorsqu'on doit s'exprimer devant un groupe. De 
"Le parcours doctoral d'Yvette représente plus concrètement l'expérience d'une francophone de milieu minoritaire qui, en dépit d'autres facteurs décisifs, à la lumière de son expérience à titre de fermme et de son bagage culturel et linguistique, associe son manque de confiance et son insécurité linguistique à de l'incompétence. Cela a altéré son expérience, et ce, en très grande partie en raison de la perception de l'Autre." plus, un déficit en matière de formation à l'intervention auprès des communautés francophones en situation minoritaire est perçu dans son récit. Le parcours doctoral d'Yvette représente plus concrètement l'expérience d'une francophone de milieu minoritaire qui, en dépit d'autres facteurs décisifs, à la lumière de son expérience à titre de femme et de son bagage culturel et linguistique, associe son manque de confiance et son insécurité linguistique à de l'incompétence. Cela a altéré son expérience, et $c e$, en très grande partie en raison de la perception de l'Autre. En somme, cet article démontre une mise en contexte d'une réalité étudiée qui met au jour les rapports sociaux qui la produit.

Comme le soutient Dewey (1983), "la portée sociale des fins et l'importance sociale des problèmes different d'un cas à l'autre " (p. 231). Des cas comme celui-ci permettent néanmoins de voir qu'au-delà de l'impact de l'insécurité linguistique sur l'expérience d'éducation de femmes, plus spécifiquement de celles inscrites aux études supérieures, cet état de fait pose un problème de représentativité non seulement pour ces dernières, mais aussi pour les membres du corps professoral et pour les francophones de milieu minoritaire en général. Il limite le potentiel d'émancipation des femmes de milieu francophone minoritaire, celui des femmes en général et celui de tous les francophones vivant en milieu minoritaire, peu importe le sexe. Cela dit, ne pas faire d'études supérieures n'est pas problématique en soi. Toutefois, nul ne peut ignorer les possibilités d'avancement qu'offrent les études supérieures, ni le statut privilégié ou le pouvoir qui y sont associés. Et le fait qu'un groupe particulier soit moins représenté et donc souvent limité - les francophones par rapport aux anglophones, mais surtout les femmes francophones en raison de la double minorisation — pose problème.

Quelques interventions pourraient mieux appuyer ces personnes dans leurs défis quotidiens. Il faudrait d'abord offrir aux femmes en milieu universitaire les services nécessaires et faire respecter des principes de bases, dont ceux proposés par Ouellette (1999). Essentiellement, chaque femme devrait avoir accès à une éducation libre de sexisme et adaptée à ses besoins, à sa socialisation et à ses projets d'avenir. Le réseau universitaire 
"Le réseau

universitaire devrait

également se

préoccuper du sexisme

systémique afin de

créer un environnement

réellement équitable

pour les deux sexes."

"...d'autres imposent

des cours qui ne

répondent pas aux

besoins des étudiants.

On y étudie, par

exemple, des romans

ou de la poésie. Ce

contenu de cours est

tout à fait louable, mais encore faut-

il reconnaître que

les étudiants ont

davantage besoin de

revoir les règles de

grammaire ou celles

entourant la rédaction

d'une dissertation

ou d'un rapport de

recherche. " devrait également se préoccuper du sexisme systémique afin de créer un environnement réellement équitable pour les deux sexes. Et ce milieu devrait aussi offrir des services complémentaires au projet pédagogique, par exemple, des services de garde pour les enfants, des services d'accompagnement, des services d'aide psychopédagogique, des réseaux de soutien institutionnalisés, des programmes de mentorat, et plus encore.

Par ailleurs, dans le but de mieux desservir les étudiants francophones provenant de milieu minoritaire et les aider à contrer l'insécurité linguistique, il faudrait offrir des cours de français qui correspondent aux besoins des étudiants francophones inscrits en première année de baccalauréat. Alors que certaines universités bilingues au pays ne dispensent aucun cours de français en première année universitaire, d'autres imposent des cours qui ne répondent pas aux besoins des étudiants. On y étudie, par exemple, des romans ou de la poésie. Ce contenu de cours est tout à fait louable, mais encore faut-il reconnaitre que les étudiants ont davantage besoin de revoir les règles de grammaire ou celles entourant la rédaction d'une dissertation ou d'un rapport de recherche. Il serait également louable d'offrir à la population étudiante un service d'appui à la rédaction, et s'il est déjà en place, de s'assurer que tous les étudiants en soient informés et que l'on accroisse ses commodités et fonctions. Une formation qui sensibiliserait à la réalité locale les professeurs œuvrant en milieu francophone minoritaire devrait également être offerte. Cela leur permettrait de mieux comprendre certains comportements sociolinguistiques et de mieux intervenir auprès de leurs étudiants. De plus, il faudrait augmenter le nombre de cours offerts en français dans les universités bilingues afin de permettre aux jeunes francophones d'étudier dans leur langue première, après les avoir sensibilisés à l'importance d'étudier en français. Enfin, on devrait inviter les orienteurs scolaires des écoles secondaires à faire la promotion des études supérieures auprès des jeunes francophones de milieu minoritaire et à renseigner ces derniers sur les avenues qui s'offrent à eux au-delà du baccalauréat.

On viserait ainsi à amenuiser - idéalement, à éliminer — les contrecoups de la double minorisation et surtout, à transmettre 
plus aisément aux communautés francophones le savoir — et bien souvent le statut, la représentation, le respect et le pouvoir décisionnel qui l'accompagnent - auquel elles ont droit.

\section{Notes}

Un pseudonyme.

2 L'intériorisation - L'identité ethnique, et les attributs qui la définissent, sont, non seulement acceptés mais intériorisé par les sujets qui ne mettent pas en cause la légitimité du discours qui les désigne par telle ou telle identité. La surenchère L'identité prescrite est non seulement acceptée et intériorisée par le sujet, mais l'aspect stigmatisant de celle-ci est mis en avant. Il s'agit d'une sorte de tentative d'assumer, et de subsumer, ses stigmates en les renforçant. Le contournement Cette stratégie est possible lorsque les contraintes sont relativement faibles, c'est-à-dire lorsque les assignations identitaires sont molles et par contre, les ressources du groupe fortes. Le retournement sémantique - L'identité minoritaire est prescrite est acceptée, avec tous les traits stigmatisés qui lui sont liés, mais ceux-ci font l'objet d'un renversement sémantique qui transforme la négativité en positivité. L'instrumentalisation de l'identité assignée — La stratégie de l'instrumentalisation est un mode d'acceptation de l'identité prescrite très différent de l'intériorisation car les acteurs ont une conscience plus claire de la nature sociale et assignée, de leur identité; s'ils l'acceptent c'est parce qu'ils sont conscients aussi de l'inégalité du rapport de forces dans lequel ils se trouvent. Mais, suivant une stratégie des faibles bien connue, ils tentent de s'en servir à leur profit. La recomposition identitaire - Cette stratégie est souvent liée à la production d'une nouvelle identité collective, née de la communauté de traitement opéré par le majoritaire, ainsi que d'une certaine communauté de destin. L'assimilation au majoritaire - Il s'agit, là aussi, d'une stratégie par essence individuelle qui consiste, pour nier, ou pour se débarrasser, d'une identité minoritaire infériorisée, à se désolidariser de son groupe d'appartenance, à refuser cette appartenance pour chercher à pénétrer dans le groupe majoritaire. Le déni-Si, dans le cas précédent, les sujets se reconnaissent dans l'identité minorisée que le regard de l'autre leur assigne et souhaitent en changer, ceux qui adoptent le déni refusent même l'idée de cette assignation : ils ne se reconnaissent pas dans l'identité qu'on leur tend. D'une certaine manière, ils refusent à l'autre le pouvoir de décider qui ils sont. L'action collective - En effet, certaines identités sont définies par des statuts sociaux trop contraignants pour que l'on puisse résoudre les contradictions ou les tensions au niveau individuel [...] En réalité, [dans de telles conditions] les identités individuelles ont peu de chances d'être valorisées si l'identité collective ne l'est pas. Inversement, l'identité collective n'est pas seulement un "modèle d'identification " proposé aux membres d'un groupe, elle est aussi le "produit » de leur action collective (Taboada-Leonetti, 1999, p. 64-77).

3 Les chiffres sont sensiblement les mêmes au Nouveau-Brunswick, où Yvette a fait ses études de baccalauréat : $12 \%$ des femmes du Nouveau-Brunswick et $11 \%$ des hommes du NouveauBrunswick de 15 ans et plus détiennent un diplôme universitaire (Conseil consultatif sur la condition de la femme du Nouveau-Brunswick, 2006).

4 L'étude originale présente six catégories, soit les éléments de réponse reliés à l'identité, à l'entourage, à l'expérience, au fait d'être femme, à l'(in)sécurité linguistique et au sens accordé à la poursuite d'études supérieures. En somme, notre méthode d'analyse ne s'est pas définie par la forme (l'hypothèse), mais par la fin (la signification) (Deschamps, 1993). 
5 Le chiac s'avère, grosso modo, un parler, voire un système linguistique, ou se mêlent des mots et des structures de la langue française et de la langue anglaise. Ce mélange vernaculaire est parlé principalement au Nouveau-Brunswick, notamment dans les régions de Moncton et Shédiac.

6 S'exprimer de manière " trop correcte ", c'est-à-dire le plus souvent d'une manière grammaticalement incorrecte du fait même de vouloir parler le plus correctement possible, et se corriger constamment, parfois pour rendre fautive une tournure qui, au départ, ne l'était pas.

\section{Bibliographie}

ALLARD, R., Kenneth DEVEAU et Rodrigue LANDRY (2009). Et après le secondaire? Étude pancanadienne des aspirations éducationnelles et intentions de faire carrière dans leur communauté des élèves de $12^{e}$ année d'écoles de langue française en situation minoritaire, Rapport de recherche, Institut canadien de recherche sur les minorités linguistiques Fondation canadienne des bourses d'études du millénaire, avec la collaboration de l'Association des universités de la francophonie canadienne.

ANGERS, Maurice (2005). Initiation pratique à la méthodologie des sciences humaines, Anjou, Éditions CEC.

BALL, Stephen et collab. (2001). «Décisions, différenciations et distinctions : vers une sociologie du choix des études supérieures ", Revue Française de Pédagogie,Vol. 136, p. 65-75.

BLAIS, Mireille, et Stéphane MARTINEAU (2006). "L'analyse inductive générale : description d'une démarche visant à donner un sens à des données brutes ", Recherches qualitatives, Vol. 26, No2, p.1-18.

BOERSMA, P. Dee, Debora GAY, Ruth A.JONES, Lynn MORRISON et Helen REMICK (1981). "Sex differences in college student-faculty interactions : Fact or fantasy? ", Sex roles, Vol. 8, $\mathrm{N}^{\circ}$ 1, p. $775-784$.

BOURDAGES, Louise (2001). La persistance aux études supérieures. Le cas du doctorat, Sainte-Foy, Les Presses de l'Université du Québec.

BOURDIEU, Pierre, et Patrick CHAMPAGNE (1992). "Les exclus de l'intérieur ", Actes de la recherche en sciences sociales, Vol. 91, N¹, p. 71-75.

BOURDIEU, Pierre (1982). Ce que parler veut dire. L'économie des échanges linguistiques, Paris, Fayard.

BOURDIEU, Pierre, et Jean-Claude PASSERON (1964). Les héritiers. Les étudiants et la culture, Paris, Minuit.

BRETEGNIER, Aude (1999). Sécurité et insécurité linguistique. Approches sociolinguistique et pragmatique d'une situation de contacts de langues : la Réunion, Thèse de doctorat, Université de la Réunion.

CALVET, Louis-Jean (1993). La sociolinguistique, Coll. Que sais-je?, Paris, PUF.

CAMERON, Deborah (1995). Verbal Hygiene, London, Routledge.

CANADA, Katherine, et Richard PRINGLE (1995). "The role of gender in college classroom interactions : A context approach ", Sociology of Education, $\mathrm{N}^{\circ} 68$, p. 161-186.

CEFAÏ, Daniel (2001). «Les cadres de l'action collective. Définitions et problèmes », sous la direction de Daniel Cefaï et Danny Trom, Les formes de l'action collective. Mobilisation dans des arènes publiques, Paris, Éditions de l'EHESS, coll. Raisons pratiques, p. 51-97. 
CHOY, Susan (2001). Students Whose Parents Did Not Go To College: Postsecondary Access, Persistence and Attainment, Washington, National Center for Education Statistics.

CONSEIL CONSULTATIF SUR LA CONDITION DE LA FEMME DU NOUVEAUBRUNSWICK (2006), Faits et chiffres, réf. du 12 août 2008, http://www.gnb.ca/0012/womensissues/wg-es/careersurf/factsfigures-f.asp\#edu.

COULON, Alain (1993). Le métier d'étudiant, Paris, PUF.

DE LANDSHEER, G. (1979). Dictionnaire de l'évaluation et de la recherche en éducation, Paris, PUF.

DESCHAMPS, Chantal (1993). L'approche phénoménologique en recherche, Montréal, Guérin.

DEWEY, John (1983). Démocratie et éducation, Paris, Armand Colin.

DEWEY, John (1968). Expérience et éducation, Paris, Armand Collin.

ENGLE, Jennifer (2007). "Postsecondary access and success for first-generation college students ", American Academic, Vol.3, No1, p. 25-48.

FRANCARD, Michel (1997). "Insécurité linguistique », sous la direction de Marie-Louise Moreau, Sociolinguistique. Concepts de base, Liège, Mardaga, p. 170-176.

FRANCARD, Michel (1993). "Trop proches pour ne pas être différents. Profils de l'insécurité linguistique dans la Communauté française de Belgique ", sous la direction de Michel Francard, L'insécurité linguistique dans les communautés francophones périphériques, Louvain-La-Neuve, Actes du colloque de Louvain-La-Neuve, p. 61-70.

HORN, Laura, et Anne-Marie NUÑEZ (2000). « Mapping the Road to College : First-Generation Students' Math Track, Planning Strategies, and Context of Support », Washington, National Center for Education Statistics.

HOWARD, Jay, et Amanda HENNEY (1998). "Student participation and instructor gender in the mixed-age college classrooom ", The Journal of Higher Education, Vol. 69, №4, p. 384-405.

JANZ, Teresa, et Sandra PYKE (2000). "A scale to assess strudent perceptions of academic climates ", The Canadian Journal of Higher Education, Vol. 30, º1, p. 89-122.

KOZANITIS, Anastassis (2001). «Classroom participation and its effect on grades ", Mextesol Journal, Vol. 24, N4, p. 33-41.

LABOV,William (1976). Sociolinguistique, Paris, Éditions de Minuit.

LECLERC, Jacques (2007). «Le multilinguisme : une source de conflits » L'aménagement linguistique dans le monde, réf. du 22 juin 2008 http://www.tlfq.ulaval.ca/axl/langues/3cohabitation_sources_

conflits.htm

$M^{C}$ CARRON, Graziella, et Karen INKELAS (2006). "The gap between educational aspirations and attainment for first-generation college students and the role of parental involvement ", Journal of College Student Development, Vol.47, N5, p. 534-549.

MOREAU, Marie-Louise (1997). Sociolinguistique. Concepts de base, Liège, Mardaga, p. 170-176.

MUJAWAMARYA, Donatille, et Christabelle SETHNA (printemps 2005). "L'Université d'Ottawa à l'égard des défis de l'équité en emploi et en éducation ", Éducation et francophonie, Vol. XXXIII, $\mathrm{N}^{\circ} 1$.

OFFICE DES AFFAIRES FRANCOPHONES (2005). Profil statistique - Les femmes francophones en Ontario, Toronto, Gouvernement de l'Ontario.

OUELETTE, Jeannine (1999). Les femmes en milieu universitaire. Liberté d'apprendre autrement, Ottawa, Les Presses de l'Université d'Ottawa. 
ROBBINS, Wendy, et M. Olivier (2006) Vérifications féministes et d'équité 2006. Indicateurs choisis pour les universités canadiennes, réf. du 4 octobre 2008, www.fedcan.ca/française/issues/ whatsnew.

ROY, Simon (2006). "L'étude de cas », sous la direction de Benoît Gauthier, Recherche sociale. De la problématique à la collecte de données, Québec, Les Presses de l’Université du Québec.

SADKER, Myra, et David SADKER (1986). «Sexism in the classroom : From grade school to graduate school ", Journal of Experimental Education, Vol. 63, №2, p. 127-138.

SAVOIE-ZAJC, Lorraine (2006). "L'entrevue semi-dirigée ", sous la direction de Benoit Gauthier, Recherche sociale. De la problématique à la collecte de données, Québec, Les Presses de l'Université du Québec.

SCHNEUWLY, Bernard (2008). «Le rapport à l'écrit : une notion - deux dimensions en interaction dynamique ", sous la direction de Suzanne G. Chartrand et Christiane Blaser, Le rapport à l'écrit : un outil pour enseigner de l'école à l'université, Namur, Presses universitaires de Namur.

SEIDMAN, Irving (2006). Interviewing as Qualitative Research. A Guide for Researchers in Education and the Social Sciences, New York et London, Teachers College Press, Columbia University.

SINGY, Pascal (2004). Identités de genre, identités de classe et insécurité linguistique, Berne, Peter Lang.

SMITH, Dorothy (1987). The everyday world as problematic: a feminist sociology, Boston, Northeastern University Press.

SMITH, Dorothy (1981). "Le parti pris des femmes ", dans Yolande Cohen, Femmes et politique, Montréal, Le Jour.

STALKER, Jackie, et Susan PRENTICE (1998). Illusion of Inclusion :Women in Post-secondary Education, Halifax, Fernwood Publishing.

SWIGGERS, Pierre (1993). "L'insécurité linguistique : du complexe (problématique) à la complexité du problème ", sous la direction de Michel Francard, L'insécurité linguistique dans les communautés francophones périphériques, Actes du colloque de Louvain-La-Neuve, Louvain-La-Neuve, p. 19-30.

TABOADA-LEONETTI, Isabel (1999). "Stratégies identitaires et minorités : le point de vue du Sociologue ", sous la direction de Carmel Camilleri, Joseph Kastersztein, Edmond Marc Lipiansky, Hanna Malewska-Peyre et Ana Vasquez, Stratégies identitaires, Paris, PUF.

THOMAS, David (2006). "A general inductive approach for analyzing qualitative evaluation data ", American Journal of Evaluation, Vol. 27, №2, p. 237-246.

TRAISNEL, Christophe, et Isabelle VIOLETTE (2010). «Qui ça, nous? La question des identités multiples dans l'aménagement d'une représentation de la francophonie en Acadie du NouveauBrunswick », sous la direction de Nathalie Bélanger, Nicolas Garant, Phyllis Dalley et Tina Desabrais, Produire et reproduire la francophonie en la nommant, Sudbury, Prise de parole, p. 101-122.

TRUDGILL, Peter (1998). «Sex and Covert Prestige », sous la direction de Jennifer Coates, Language and Gender: A Reader, Oxford, Oxfort University Press, p. 21-28.

TRUGILL, Peter (1972). «Sex, Covert Prestige and Linguistic Change in the Urban British English of East Anglia ", Language in Society,Vol.1, p. 179-195.

UNIVERSITÉ D'OTTAWA (2007). «Faits en bref. Inscriptions (automne 2007) » réf. du 22 juin 2008, http://web5.uottawa.ca/mcs-smc/faitsenbref/inscriptions.html.

VEZINA,Vande Jane (1998). «It's Still a Man's World :Women and Graduate Study », dans The Illusion of inclusion:Women in Post-Secondary Education, sous la direction de Jackie Stalker et Susan Prentice, Halifax, Fernwood Publishing, p. 72-84. 